\title{
Printed Coupled Loop Wideband Antenna Design for Wireless Communication
}

\author{
Shoaib Ahmed Khan \\ COMSATS Institute of Information and Tech, Abbottabad, Pakistan
}

\begin{abstract}
This paper explains the wideband loop antenna design for wireless communication. Printed antennas are supposed to be more attractive for mobile phone operations for achieving WWAN purposes. In mobile phones theses antenna usually cover the internal circuitry at the top or bottom of the device and they also require further isolation in order to get negligible coupling effect properly that comes due to the coupling between the radiation part of the antenna and the ground plane [1] to [3]. This will limit the internal integration of the antenna with the other circuitry of the cell phone for using it for WWAN purposes for example for achieving better SAR [4]. The proposed antenna consists of the folded loop and some patches in the ground plane to achieve desired goals. The ground plane and feeding part of the antenna are separated by FR4 substrate. Input is given through the capacitively coupled feeding port on the backplane. The antenna is designed to cover ranges of many bands such as, GSM (850/900MHz and 1800/1900MHz), WLAN (2.4GHz) and also the higher frequency bands such as ISM band. The antenna designed, gives very good return loss and has fine radiation pattern. The simulation of antenna in software called HFSS, version 11.0. I personally prefer to design antenna on this software because it gives a lot of customization options.
\end{abstract}

\section{Introduction}

Nowadays the growing scientific research has made life dependent on new technology. The blessing of science has made living quite easy and flexible. First when mobile phone was invented, human became able to make contact with anyone from any corner of the world but with the invention of smart phones and laptops, we became able to carry on our daily updates related to news, weather, accessing our online accounts and even we can now carry on our business while we are on move. Now in this paper an attempt has been made to share the innovative idea in the field of antenna and telecommunication.

From current situation of growing rate of the use of smart phones, has increased the demands for evolution in antenna designing. In smart phones generally PIFA or patch antenna is used. Smart phones usually require multiple bands feature for its various functions and in case of patch antenna, generally a separate antenna is used for each band which are the integrated together to achieve multiple bands. Now in this case, a folded loop antenna has been used which will cover all the desired frequency bands.

The proposed antenna is for handheld devices and will operate on GSM/PCS/UMTS/WLAN bands. The proposed prototype of antenna will be of the folded loop structure and will be suitable to be fitted in a smart phone or in any other device depending on radio technology. The designed antenna will radiate so that its $1^{\text {st }}$ band will occur from $0.85 \mathrm{GHz}$ to $2.8 \mathrm{GHz}$ with the bandwidth of $1.95 \mathrm{GHz}$ and the $2^{\text {nd }}$ band from $3.6 \mathrm{GHz}$ to $4.9 \mathrm{GHz}$ occupying the bandwidth of $1.3 \mathrm{GHz}$. The dimensions of the antenna are $116 \mathrm{~mm} \times 60 \mathrm{~mm} \times 10 \mathrm{~mm}$. All the simulated and experimental results are shown below in the further description.

\section{Antenna Design}

The antenna is designed for handheld devices such as smart phones or tablets. At the very bottom we will be having a ground plane, upon which FR4 substrate is used and on the other side of the FR4, coupling strip and feeding strip, which are capacitively coupled. 


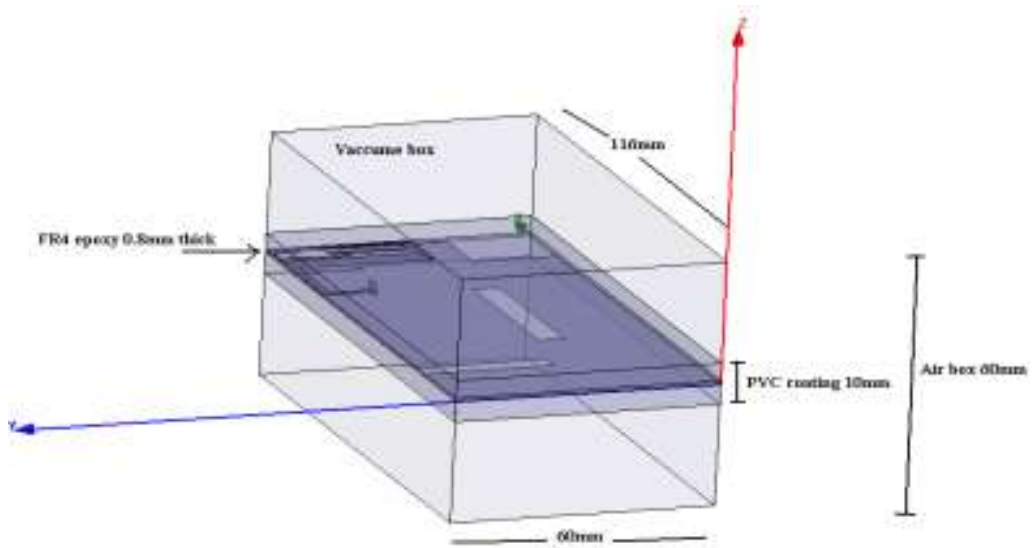

Figure 1: $3 \mathrm{~d}$ view of the simulated antenna

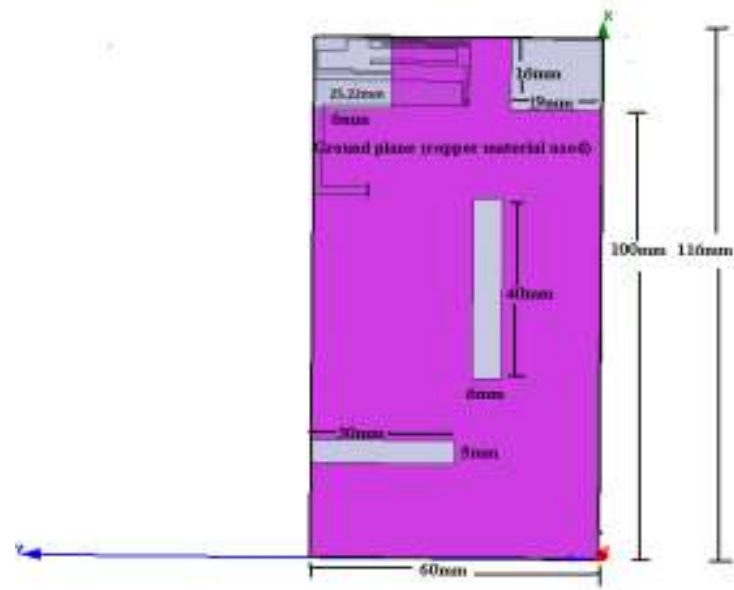

Figure 2: Ground plane

Starting from the ground plane, the total dimensions of the ground plane are $100 \mathrm{~mm} \times 60 \mathrm{~mm}$ and its protruded part of the ground plane is $16 \mathrm{~mm} \times 15.78 \mathrm{~mm}$. There are also no-ground plane regions on the both sides of the protruded ground plane, one with the dimensions of $16 \mathrm{~mm} \times 25.22 \mathrm{~mm}$ and other with $16 \mathrm{~mm} \times$ $19 \mathrm{~mm}$. The no-ground planes are defined to allow some free space for other components of antenna. The protruded ground plane improves overall efficiency of antenna. The dimensions of the FR4 substrate are $116 \mathrm{~mm}$ $\mathrm{x} 60 \mathrm{~mm}$ and thickness of $0.8 \mathrm{~mm}$ with relative permittivity of 4.4 . There are two strips on the antenna both are on designed over the FR4, the feeding strip and coupling strip. When input is applied at the feeding port of antenna having input resistance of $50 \Omega$, gives better resonance specially at the lower bands of GSM[5].The coupling strip is shortened with the ground plane via cylinder. The input energy to antenna is given via feeding port located on the end of the feeding strip. In order to increase the electrical length of the antenna, the loop of the antenna has been folded many times. The antenna can also be coated with PVC of thickness $10 \mathrm{~mm}$ to make it suitable for use in smart phone.

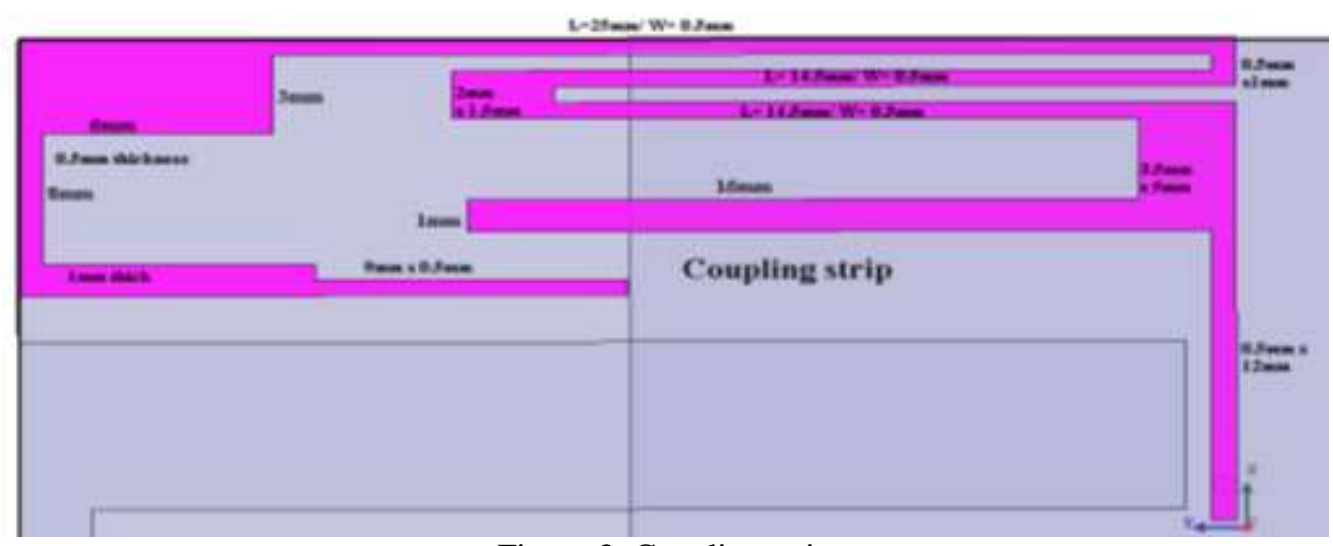

Figure 3: Coupling strip 


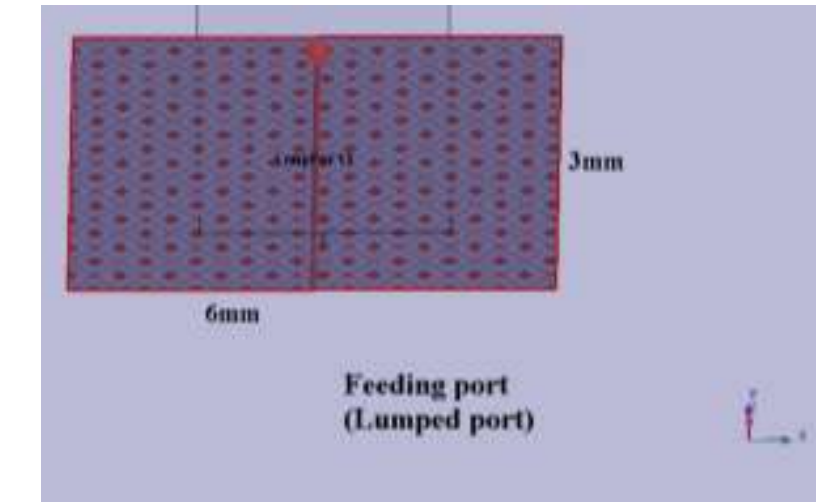

Figure 4: Feeding port

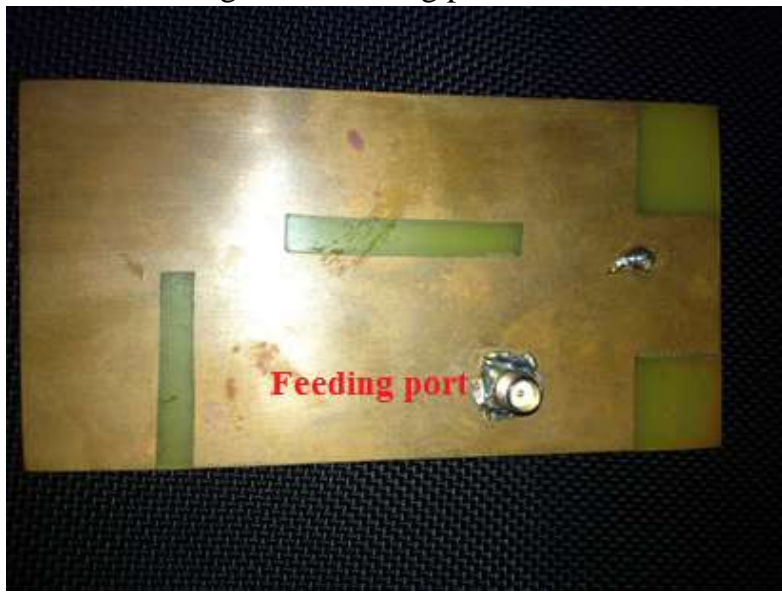

Figure 6: Hardware image (Ground plane)

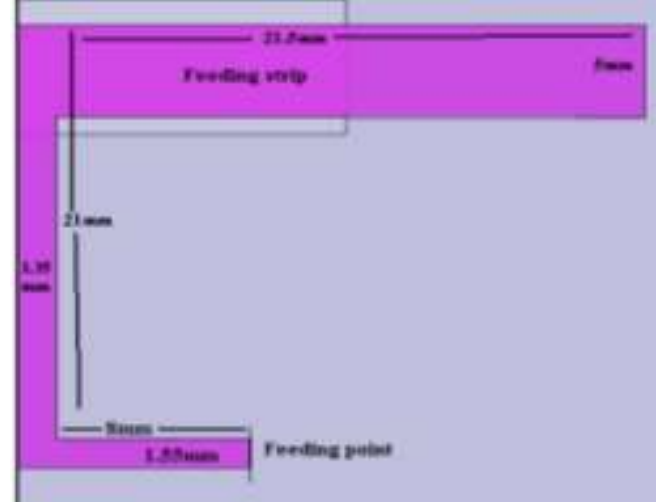

Figure 5: Coupling strip

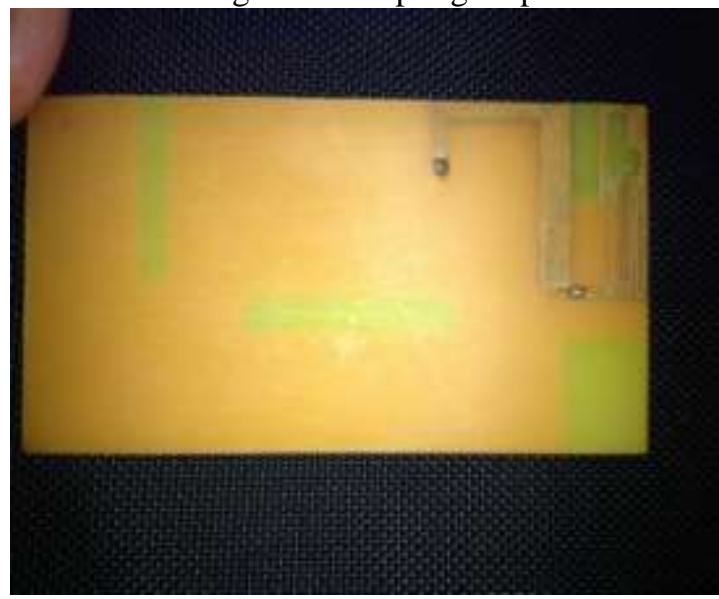

Figure 7: Resonating part of antenna

There are also two rectangular slots cut in the ground plane. One slot is with the dimensions of $40 \mathrm{~mm} \mathrm{x}$ $6 \mathrm{~mm}$ and other slot with dimensions of $5 \mathrm{~mm} \times 30 \mathrm{~mm}$. These slots helped in improving the return loss and increased the electrical length of the antenna.

\section{Results}

The proposed antenna has been designed and simulated in Ansoft HFSS. Then after simulation, antenna was fabricated and tested in Telecommunication Lab of COMSATS Institute of Information and Tech, Abbottabad, Pakistan. And the measured results were taken using network analyzer. The simulation and the measured results are shown below in figure.

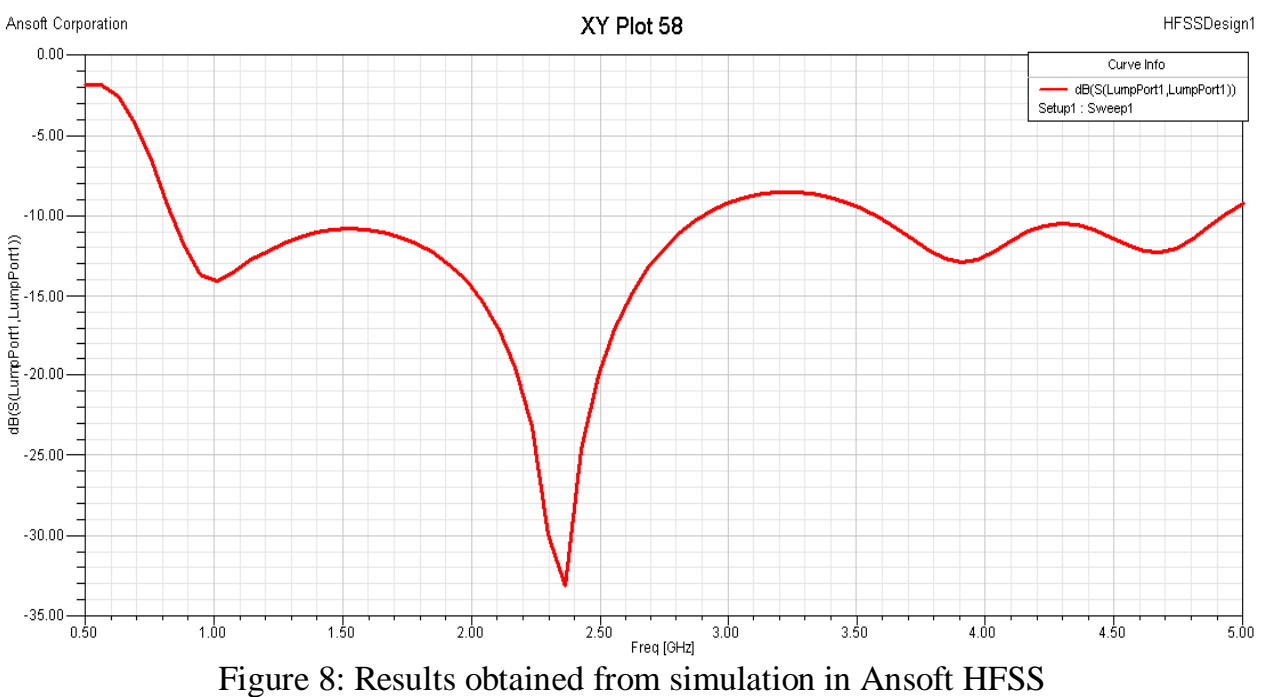




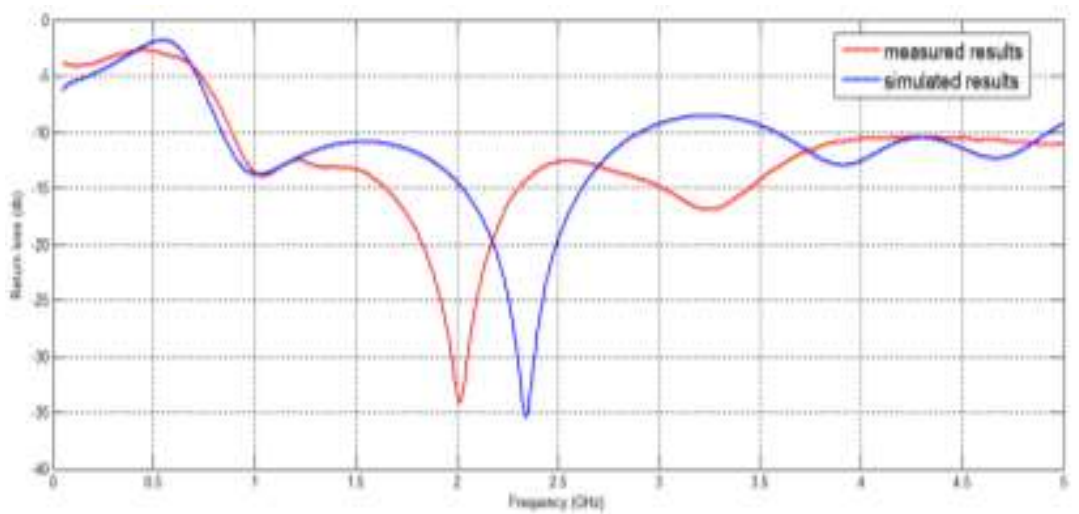

Figure 9: Comparison of both simulated results (one with blue color) and the measured results (one with red color)

In the measured results the graph has been shifted little on left, this is because of the coaxial cable used for taking readings was not properly covered, which resulted in effecting the lower frequencies. The antenna is covering GSM/UMTS/WWAN/PCS/ISM. The input impedance is set $50 \mathrm{ohm}$ to achieve the mentioned goals. From results it is quite clear that the antenna is covering two frequency bands, the first one is an Ultra wide band and the second one is wide band. The $1^{\mathrm{ST}}$ band Is starting from $0.85 \mathrm{Ghz}$ to $2.8 \mathrm{GHz}$ which Is covering all GSM/UMTS/PCS/WWAN bands and the $2^{\text {nd }}$ band is starting from $3.6 \mathrm{Ghz}$ to $4.9 \mathrm{GHz}$ which is basically covering ISM band. The radiation pattern and the gain of the antenna are giver below.
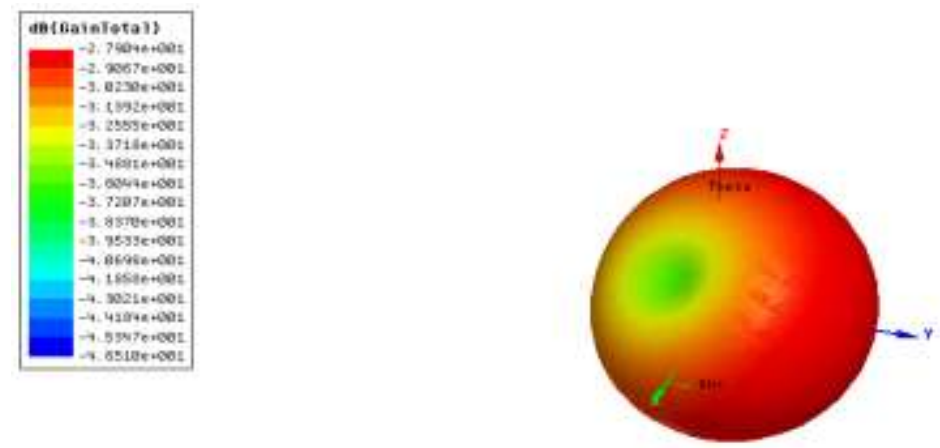

Figure 10: Total gain of antenna
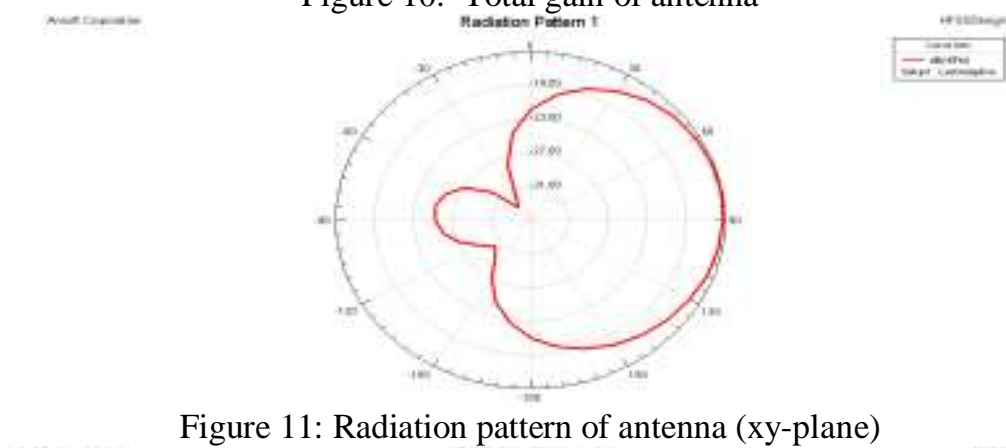

Figure 11: Radiation pattern of antenna (xy-plane)
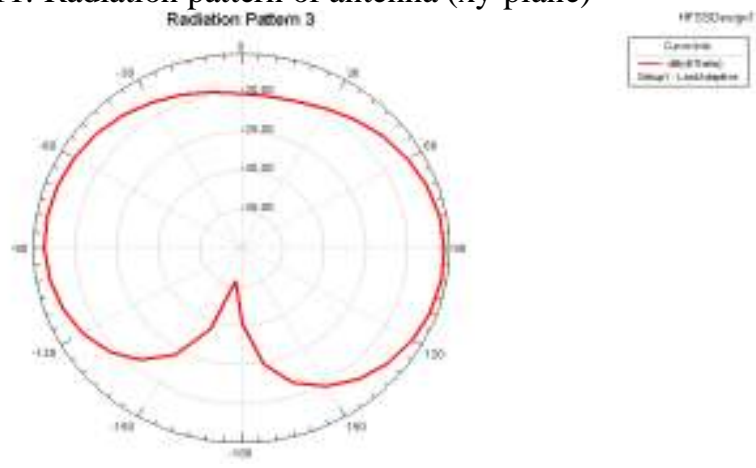

Figure 12: Radiation pattern of antenna (yz-plane) 

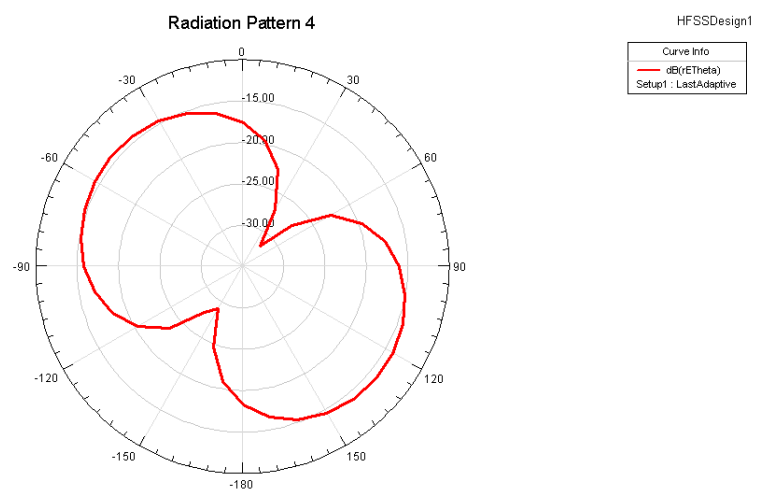

Figure 13: Radiation pattern of antenna (xz-plane)

\section{Conclusion}

First of all it concluded that loops antenna is a very good choice for smart phones or other devices working in the frequency range of this antenna. By using the technique of the folding the loop of antenna, you can get more electrical length by maintaining the overall size of antenna constant. The protruded ground plane plays an important role in the getting better return loss and better gain. By cutting the parts of ground plane we can increase the electrical size of antenna and can also get the better return loss.

From results it is quite clear that this antenna can be used in almost all the wireless devices that operate on frequency range of $850 \mathrm{MHz}$ to $2.8 \mathrm{GHz}$ or from $3.6 \mathrm{GHz}$ to $4.9 \mathrm{GHz}$. This is a cost effective antenna and suits best for the handheld devices.

\section{References}

[1] C. M. Su, K. L. Wong, C. L. Tang and S. H. Yeh, "EMC internal patch antenna for UMTS operation in a mobile device," IEEE Trans. Antennas Propagat., vol. 53, pp. 3836-3839, 2005.

[2] K. L. Wong and C. H. Chang, "Surface-mountable EMC monopole chip antenna for WLAN operation," IEEE Trans. Antennas Propagat., vol. 54, pp. 1100-1104, 2006.

[3] C. H. Wu, K. L. Wong and J. S. Row, "EMC internal GSM/DCS patch antenna for thin PDA phone application," Microwave Opt. Technol. Lett., vol. 49, pp. 403-408, 2007.

[4] American National Standards Institute (ANSI), "Safety levels with respect to human exposure to radiofrequency electromagnetic field, $3 \mathrm{kHz}$ to $300 \mathrm{GHz}, "$ ANSI/IEEE standard C95.1, 1999.

[5] Y. W. Chi and K. L. Wong, "Quarter-wavelength printed loop antenna with an internal printed matching circuit for GSM/DCS/PCS/UMTS operation in the mobile phone,” IEEE Trans. Antennas Propagat., vol. 57, pp. 2541-2547, 2009. 